\title{
On Divisibility By Nine of the Sums of Even Amicable Pairs
}

\section{By Elvin Lee}

\begin{abstract}
Most known even amicable pairs have sums divisible by nine [9]. The general form of the exceptions to the rule of divisibility by nine (Gardner's rule) is deduced and the results expressed in the form of a theorem. A computer search based on a corollary to the theorem is described and six new exceptions to Gardner's rule are found.
\end{abstract}

An amicable number pair is a pair of numbers, $M, N$, satisfying

$$
\sigma(M)=M+N=\sigma(N),
$$

where $\sigma$ denotes the divisor sum function [1]. More than 550 amicable pairs are known in which both members of the pair are even. Surprisingly the sums of most if not quite all of these pairs are divisible by nine. The exceptions are so few that it is easy to be misled into believing that all even amicable pairs possess this property, a belief which is confirmed when one considers some simple special cases. For instance, consider the pairs $M=2^{n} p q, N=2^{n} r$ where $p, q, r$ are primes given by the formula of Thabit-ben-Korrah [1].

$$
p=3 \cdot 2^{n-1}-1, \quad q=3 \cdot 2^{n}-1, \quad r=9 \cdot 2^{2 n-1}-1 .
$$

Here $M+N=2^{n}(p q+r)=9 \cdot 2^{n} \cdot\left(2^{2 n}-2^{n-1}\right)$.

It is instructive to next consider a somewhat more general case including the above since it requires a lemma which we shall need later anyway. Let $M, N$ be given by

$$
M=E p q, \quad N=E r,
$$

where $E$ is an even common factor of $M$ and $N$ and where the distinct primes, $p, q, r$ do not divide $E$. From (1) and (3) we get

$$
r=p q+p+q,
$$

and since $p$ and $q$ are distinct we take $q>p$. By virtue of the following lemma, $p>3$ and we need consider only primes of forms $6 n \pm 1$.

Lemma I. Neither member of an even amicable pair is divisible by three.

Proof. Both $M$ and $N$ may not be $\equiv 0 \bmod 3$ since they would then both be abundant, contradicting the fact that the greater member of an amicable pair is deficient. Thus, we may write

$$
M=2^{r} 3^{t} c, \quad N=2^{s} b .
$$

Since $N \not \equiv 0 \bmod 3,(1)$ and (5) give

$$
\sigma(M)=M+N=\sigma(N) \equiv 1,2 \bmod 3 .
$$

Because of (6) $r$ and $s$ must both be even so that $M=2^{2} \cdot 3 \cdot k$ and $N=2^{2} \cdot l$ where $k \geqq 1$ and $l>3 k$ since $M$ must be abundant, making $M<N$. For $k \geqq 1, M / \sigma(M) \leqq$

Received October 9, 1968. 
$3 / 7$ and for $l>3 k, N / \sigma(N)<4 / 7$ contradicting (1) which implies $M / \sigma(M)+$ $N / \sigma(N)=1$. Thus our lemma is proven and we continue with our consideration of (3).

Both $p$ and $q$ in (3) may not be of the form $6 n+1$ since by (4) $r$ would not be a prime. From (1), (3), and (4) we have

$$
\sigma(E) / E=(2 p q+p+q) /(p q+p+q+1) .
$$

If one of $p, q$ is of form $6 n+1$ and the other of form $6 n-1, E \equiv 0 \bmod 3$ since 3 cannot cancel out of the denominator when (7) is reduced to lowest terms but this contradicts Lemma I so $p$ and $q$ are therefore both of form $6 n-1$. But then $\sigma(N)=$ $\sigma(E) \cdot(p+1)(q+1) \equiv 0 \bmod 9$ and therefore $M+N \equiv 0 \bmod 9$ so that we have proven that all even amicable pairs of form (3) have sums divisible by 9 .

We now take up the general case and prove the following theorem.

Theorem I. The sums of all even amicable pairs $M, N$ are divisible by nine with the exception of the following three forms:

$$
\begin{aligned}
& \text { (a) } M=2^{e} \prod B \prod C, \quad N=2^{e^{\prime}} \prod B^{\prime} \prod_{o} C^{\prime}, \quad M+N \equiv 2 \bmod 3, \\
& \text { (b) } M=2^{e} A \prod B \prod C, \quad N=2^{e^{\prime}} D \prod B^{\prime} \prod C^{\prime}, \quad M+N \equiv 0 \bmod 3, \\
& \text { (c) } M=2^{o} \prod B \prod C, \quad N=2^{e} D \prod B^{\prime} \Pi C^{\prime}, \quad M+N \equiv 0 \bmod 3,
\end{aligned}
$$

where $A, B, C, D$ are factor types defined in Table $\mathrm{I}$, o and e refer to odd and even respectively, $\Pi$ denotes the product of an unspecified number of terms of the type indicated unless subscripted o when it refers to an odd number, and the primes distinguish exponents and factors in $N$ which are not necessarily all distinct from corresponding quantities in $M$.

Proof. In consequence of Lemma I the odd prime factors of $M, N$ are all of forms $6 j \pm 1$. Table I summarizes the modulo three residue properties of the possible factors of $M, N$. We distinguish three cases in our proof according to the parities of $r$ and $s$ in the following expression for the general even amicable pair

$$
M=2^{r} a, \quad N=2^{s} b .
$$

Case I. $r$ odd, s odd. We have $\sigma(M)=\sigma(N) \equiv 0 \bmod 3$ which implies $M+N \equiv$ $0 \bmod 3$. But since $2^{r}$ and $2^{s} \equiv 2 \bmod 3$ one of $M, N$ must contain an odd number of factors of type $A$. Suppose it to be $M$. Then $\sigma(M) \equiv 0 \bmod 9$ implying $M+N \equiv$ $0 \bmod 9$.

Case II. $r$ odd, s even. If $r \equiv 5 \bmod 6, \sigma\left(2^{r}\right) \equiv 0 \bmod 9$, implying $M+N \equiv 0$ $\bmod 9$. Otherwise we have $\sigma\left(2^{r}\right) \equiv 0 \bmod 3$ and $\sigma\left(2^{s}\right) \equiv 1 \bmod 3$. From Table I it is seen that $N$ must have either

(a) A factor of type $A$ in which case either $M$ or $N$ must have at least another factor of type $A$ to make $M+N \equiv 0 \bmod 3$. But then $\sigma(M)$ or $\sigma(N)$ as the case may be is $\equiv 0 \bmod 9$ implying $M+N \equiv 0 \bmod 9$.

(b) A factor of type $D$ in which case $\sigma(M)=\sigma(N)=M+N \equiv 0 \bmod 3$ but not $\equiv \bmod 9$ so long as any additional factors of $M N$ are of types $B$ and $C$ only.

Case III. $r$ even, s even. Since $2^{r}, 2^{s}, \sigma\left(2^{r}\right)$ and $\sigma\left(2^{s}\right)$ are all $\equiv 1 \bmod 3$ we see from Table I that

(a) If $M N$ contains factors of type $A$ the total number, $l$, of these factors must 
be odd. $l>1$ implies $M+N \equiv 0 \bmod 9$ since one of $M, N$ then contains at least two factors of type $A$. If $l=1$ we are at liberty to assign the type $A$ factor to $M$ so that $\sigma(M) \equiv 0 \bmod 3$. Then $N$ must contain a type $D$ factor to make $\sigma(N) \equiv 0$ $\bmod 3$. If $N$ contains only one type $D$ factor then $M+N \equiv 0 \bmod 3$ but not $\equiv 0 \bmod 9$.

(b) If $M$ and $N$ contain only factors of types $B$ and $C$ we see from Table I that they must each contain an odd number of factors of type $C$ in order to satisfy (1). Then $M$ and $N$ are each $\equiv 1 \bmod 3$ and $\sigma(M), \sigma(N)$ and $M+N$ are each $\equiv 2$ $\bmod 3$.

\begin{tabular}{ccccc} 
& \multicolumn{4}{c}{ TaBLE I } \\
Form & Power & Residue & Residue of Divisor Sum & Type \\
2 & $2 l-1$ & 2 & 0 & \\
2 & $2 l$ & 1 & 1 & \\
$6 j-1$ & $2 l-1$ & 2 & 0 & $A$ \\
$6 j-1$ & $2 l$ & 1 & 1 & $B$ \\
$6 j+1$ & $3 l-2$ & 1 & 2 & $C$ \\
$6 j+1$ & $3 l-1$ & 1 & 0 & $B$ \\
$6 j+1$ & $3 l$ & 1 & 1 & $B$
\end{tabular}

Cases I-III are exhaustive and Theorem $I$ is thus proven. It remains to find amicable pairs of types (b) and (c) of Theorem I. We shall show that some type (a) pairs may be found by considering the following special consequence of Theorem $\mathrm{I}$. Our calculations were done using the CDC 1604 computer at ORNL.

Of the more than 550 known even amicable pairs [2]-[8] the majority can be represented by

$$
M=E \prod_{j} p_{i}, \quad N=E \prod_{k} q_{i}, \quad j \geqq 2, \quad k \geqq 1,
$$

where $E$ is even and relatively prime to the $p_{i}$ and $q_{i}$. The $p_{i}$ are distinct primes and the $q_{i}$ are distinct primes but the $p_{i}$ are not necessarily all different from the $q_{i}$. Pairs (9) may belong to type (a) and (cf. Case III) we have the following Corollary to Theorem I.

Corollary I. All even amicable pairs (9) have sums divisible by 9 unless $E$ and $\sigma(E)$ are $\equiv 1 \bmod 3, j$ and $k$ are both odd and the $p_{i}$ and $q_{i}$ are all of form $6 n+1$.

Thus even amicable pairs whose sums are not divisible by 9 are rather exceptional and perhaps not easily found. Since the exhaustive searches begun by Ore et al. [6] are being continued by Bratley and McKay [8], [9] we confine ourselves here to what appear to be the simplest possibilities, namely the cases $j=3, k=1$, and $j=k=3$ of $(9)$.

Letting $p, q, r \cdots$ stand for the $p_{i}, q_{i}$ of (9) we have in the first case

$$
M=E p q r, \quad N=E s .
$$

Using method II of [7] we have determined that there are no solutions of (10) with the desired properties for $E<10^{5}$. From (1) and (10)

$$
\sigma(E) / E=(p q r+s) /(s+1)
$$




$$
s=(p+1)(q+1)(r+1)-1 \text { and } M<N .
$$

For all primes $p, q, r, s$ of form $6 n+1$ such that $p q r<10^{6}$, we have computed and examined the ratios $\sigma(E) / E$ and we find that there are no solutions of (11). Combining results there are no even pairs (10) such that $M+N \not \equiv 0 \bmod 9$ for $M<10^{11}$.

In the second case we note that Corollary I admits the possibility that one of the $p_{i}$ may $=$ one of the $q_{i}$ in (9) and we find that pairs 86, 87, 94 of [2], all found by $P$. Poulet, are the only even amicable pairs previously reported whose sums are not divisible by nine. We list eight new pairs $M=E$ epr, $N=E s t u$ with $M+N \not \equiv$ $0 \bmod 9$

$$
\begin{aligned}
& 2^{4} \cdot 19 \cdot 6451 \cdot 27103, \quad 2^{4} \cdot 109 \cdot 307 \cdot 103231, \\
& 2^{4} \cdot 19 \cdot 31723 \cdot 61879, \quad 2^{4} \cdot 97 \cdot 439 \cdot 910519, \\
& 2^{6} \cdot 139 \cdot 991 \cdot 48649, \quad 2^{6} \cdot 139 \cdot 4339 \cdot 11119, \\
& 2^{6} \cdot 151 \cdot 433 \cdot 392599, \quad 2^{6} \cdot 151 \cdot 7549 \cdot 22567, \\
& 2^{6} \cdot 157 \cdot 379 \cdot 1097743, \quad 2^{6} \cdot 157 \cdot 2887 \cdot 144439, \\
& 2^{6} \cdot 271 \cdot 163 \cdot 11923, \quad 2^{6} \cdot 271 \cdot 541 \cdot 3607, \\
& 2^{6} \cdot 523 \cdot 109 \cdot 192463, \quad 2^{6} \cdot 523 \cdot 367 \cdot 57529, \\
& 2^{6} \cdot 751 \cdot 79 \cdot 799063, \quad 2^{6} \cdot 751 \cdot 2239 \cdot 28537 .
\end{aligned}
$$

The smallest known even amicable pair whose sum $\not=0 \bmod 9$ is Poulet's pair 666030256,696630544 , but to prove that this is the smallest such pair would require a rather time-consuming search.

Martin Gardner has noted [10] that the members of Poulet's pairs all have digital roots $=7$. This is also true of the six new pairs we have found. It is easy to see that this could not be the case however for pairs (10) if any exist, but, for pairs of the type found, $M$ and $N$ must be both $\equiv$ either 1,4 , or 7 modulo 9 .

Oak Ridge National Laboratory

Oak Ridge, Tennessee 37830

1. O. Ore, Number Theory and Its History, McGraw-Hill, New York, 1948, pp. 89-99. MR 10, 100 .

2. E. B. Escotт, “Amicable numbers,” Scripta Math., v. 12, 1946, pp. 61-72. MR 8, 135.

3. P. Poulet, "43 new couples of amicable numbers," Scripta Math., v. 14, 1948, p. 77.

4. M. Garcia, "New amicable pairs," Scripta Math., v. 23, 1957, pp. 167-171. MR 20 \#:51:58.

5. H. L. Rolf, "Friendly numbers," Amer. Math. Monthly, v. 72, 1965, p. 455.

6. J. Alanen, O. Ore, \& J. Stemple, "Systematic computations on amicable numbers," Math. Comp., v. 21, 1967, pp. 242-245. MR $36 \# 5058$.

7. E. J. LeE, "Amicable numbers and the bilinear diophantine equation," Math Comp., v. 22, 1968, pp. 181-187. MR 37 \#142.

8. P. Bratley \& J. MCKAY, "More amicable numbers," Math. Comp., v. 22, 1968, pp. 677678. MR 37 \#1299.

9. M. GARDNER, "A short treatise on the useless elegance of perfect numbers and amicable pairs," Sci. Amer., v. 218, no. 3, 1968, pp. 123-124.

10. M. Gard Ner, Private communication. 